\title{
TFR: A Tourist Food Recommender System based on Collaborative Filtering
}

\author{
Neda Rajabpour \\ Computer Group, \\ Shahid Bahonar \\ University, Kerman, \\ Iran
}

\author{
Amirmahdi \\ Mohammadighav \\ am \\ Computer Group, \\ Shahid Bahonar \\ University, Kerman, \\ Iran
}

\author{
Ali Naserasadi \\ Computer Group, \\ Zarand Higher \\ Education Complex, \\ Zarand, Iran
}

\author{
Majid Estilayee \\ Technical and \\ Engineering, Payam- \\ e Nour, Tehran, Iran
}

\begin{abstract}
A Nowadays using recommender systems (systems that help you to choose something) is so widespread that we can say their usage is one of the most vital necessities of human being. These systems have been made to help the users to choose the best alternative on the basis of their preferences. On the other hand, in the tourism industry, as one of the most profitmaking industries, most tourists are not familiar with the foods of the countries that they have travelled to, so it is possible that they choose a kind of food that they don't like or it is dangerous for their health because of possible disease that they suffer from. In this paper, a system is proposed for solving this problem of tourism industry, called TFR. The purpose of this system is to recommend foods to tourists according to their preferences. Moreover, TFR is able to recommend a special food to a tourist in case he/she has a special diet. To evaluate the presented system which is based on collaborative filtering, it has been used by some real users. The results show that the accuracy of TFR is $86.3 \%$, indicating the suitable efficiency of the system.
\end{abstract}

\section{General Terms}

Artificial Intelligence, Recommender System

\section{Keywords}

Food Recommender System, Tourist Recommender System, Collaborative Filtering, Content-Based Filtering

\section{INTRODUCTION}

In today's world, the volume of data is highly increasing. The existing data on the internet is one of the reasons of this explosive increase. According to Turban, internet data increase by $10 \%$ every month [1]. Among all this information, we need some devices to identify the user's interests and to present recommendations according to their interests in different situations. A type of these devices is recommender systems. These systems were invented to identify the users' interests and to help them choosing the best alternative. There were made to give recommendations to users in different aspects like movies [2,3], news [4,5], e-commerce [6], tourist [7] and some other cases.

Nowadays tourism industry is an issue that researchers pay lots of attention to, and they are trying to create different ways to solve the problems of this industry's users known as tourists [8, 9 and 10]. In 2013, about 1.087 billion touristy trips took place the world. Compared to 2012, it has grown by $5 \%$. Moreover in 2012 this industry has financially grown to $\$ 1.07$ trillion, compared to 2011, this growth was 4\% [11]. The statistics show that tourism industry is one used by a lot of users; therefore establishing efficient intelligent systems in this field could be so vitalistic.

One of the problems that tourists encounter is not having enough knowledge to choose foods in the countries they journeyed to. The use of recommender systems is one of the solutions of this problem. Overall, recommender systems in the field of food can be categorized into two groups:

Food Recommender Systems: The purpose of these systems is to recommend a kind of food to users that match their cuisine by identifying the users' preferences [12,13]. In some occasions, there are some systems recommending users a kind of food that's not harmful for their health by receiving some information about his/her diseases [14, 15].

Restaurant Recommender Systems: these systems are trying to identify some parameters (such as expenses, distance, cuisine, etc.) that are important for the users and then based on these parameters, they recommend the restaurant which correspond more to their preferences. The restaurant recommender systems are can be used by individuals or groups. Group recommender systems give suitable recommendations for all group members by identifying the whole group members' preferences $[16,17]$.

On the other hand, the people who travel to other countries as tourists face two problems:

They probably do not have much knowledge about the foods served in those countries and when they look through the restaurant's menu, they cannot choose the food matching their cuisine because they do not know anything about the foods' ingredients.

If the user follows a special diet, since he/she does not know anything about the food served in that restaurant, he/she may choose something that is harmful for him/her.

A Food Recommender System for tourists, called TFR, is presented in this paper. The presented system is web-based using a collaborative filtering to give recommendations to the users. The TFR system identifies the users' interests to help them to find their favorite food in the country they have traveled to. Moreover the system identifies harmful foods for the user if he/she has a special disease, and after omitting the harmful items, the system recommends his/her favorite food that's harmless. To the best of our knowledge, this is one of the first recommender systems in the field of tourism industry with the above approach.

The algorithm of recommending to users is adoptive. The procedure to give recommendation is as follows: first the system uses the neighbors' concept ( the neighbors' 
preferences and interests which are the same as the user) to give an accurate recommendation because the system does not have enough information about the user, but as time passes and the system gets more familiar to the user's interests and preferences, the user's own information is more effective in giving a suitable recommendation because of the changes in the adoptive algorithm's parameters.

The paper is organized as follows. In the next section some related works in the field of recommender systems will be mentioned. In the third section, the presented system and the used algorithm will be introduced. In the fourth section, the accuracy and efficiency of the system is assessed and the conclusion will be mentioned in the fifth section.

\section{PREVIEW AND RELATED WORKS}

In this section, first recommender systems are introduced and then their used techniques are assessed. Since presented subject is recommending food to tourists, first some tourists systems, and then some food systems are assessed.

\subsection{Recommender Systems}

Recommender systems help their users to choose something by identifying their preferences, and recommend items based on their interests and necessities. To identify the user's preferences, explicit and implicit methods are used [18, 19]. These systems are divided into individual, and group systems. In the individual method, a recommendation is given based on one user's preferences, but in group method, all group members' preferences are paid attention to $[2,3]$.

Filtering method is one of the most important parts of the recommender system. Some of the most famous filtering methods are:

- Content-based Filtering:In this method, the system recommends to the user based on the present user's information and interests. His/her selections and experiences in the past are paid attention to, and the given recommendations are more similar to the items already selected by the user [20,21].

- Collaborative Filtering: In this method, the recommended items are given according to other people who have the same interests to the target user[22].

- Hybrid Filtering: In this method, two or more filtering methods are mixed to give more suitable recommendations to the users[23].

We can mention the cold-start issue among all the problems that recommender systems encounter. This problem takes place when there is not enough information in the system in order to recommend. To solve this problem, lots of suggestions have been presented up to now $[24,26]$.

\subsection{Tourist Recommender Systems}

Many studies have been done about recommender systems in which they tried to give suitable recommendations to tourists according to their preferences. These tourist systems help the tourists in various aspects. In the below, some of these systems will be studied:
In [7] a hybrid recommender system is presented, developed on cell phones. The system recommends the most likely interesting places according to the user's present location and their interests. In this system 3D GIS architecture is used. About the places the user is far from, the system displays the distance and the way to get to that place to the tourist. In [8] it is mentioned that mobile recommender systems in tourism aspect do not have the ability to extract information, and evaluate the rating done by other tourists with the same interests; therefore, Itravel recommender system has been presented which uses the collected information of other users to give a suitable recommendation to the target user. Peer to peer mobile communications are used (Wi-Fi or Bluetooth) to exchange the tourists' ratings via their cell phones. This is financially economical. In paper [27] it is mentioned that since there are lots of places which users can choose to travel and there are also lots of information, it's difficult for users to make a decision in this case (situation), therefore, a system has been developed to introduce the most suitable places to them.

Contrary to the mentioned systems, in [28] it is tried to present a system to be able to give recommendations to a group of people in addition to give individual recommendations. The system can give a list of the activities which are possible for tourists to do in a city, and also a tour schedule of activities, the distance between places and their timetable.

\subsection{Food Recommender Systems}

As mentioned earlier, food recommender systems fall into two categories: Restaurant recommender systems and the systems which recommend the food based on the users' preferences and possible diet.

In [29] a Hybrid recommender system is presented using knowledge-based and collaborative filtering system. In this system GIS and Google-map are used to present suitable recommendations to the users. In [17] a pocket restaurant detector is presented whose purpose is to recommend a restaurant to a group of people. This recommendation is based on people's location and interests. Each member of the group makes a list of his/her preferences including expenses, distance, cuisine, and amenities. Then the system give recommendations to the group based on their preferences and their significance to each member of the group. In [16], a mobile application presented to identify the restaurants which are near the device. It has been mentioned since users often face short of time; this system draws out notable points about these restaurants given by device reviewers and summarizes them.

Another group of recommender systems try to recommend to users based on their diets. In [14] it is tried to provide a personal diet for the patient based on his favorite foods. In [15] a healthy diet system based on data mining implemented in which first they receive some information about people's diets then by the help of data mining, useful foods are recommended to them. In [12] it is stated that today online supermarkets are dead places where users only notify how much or how many of a product they want; therefore, a new approach for making an online store based on recommending diets has been presented. 


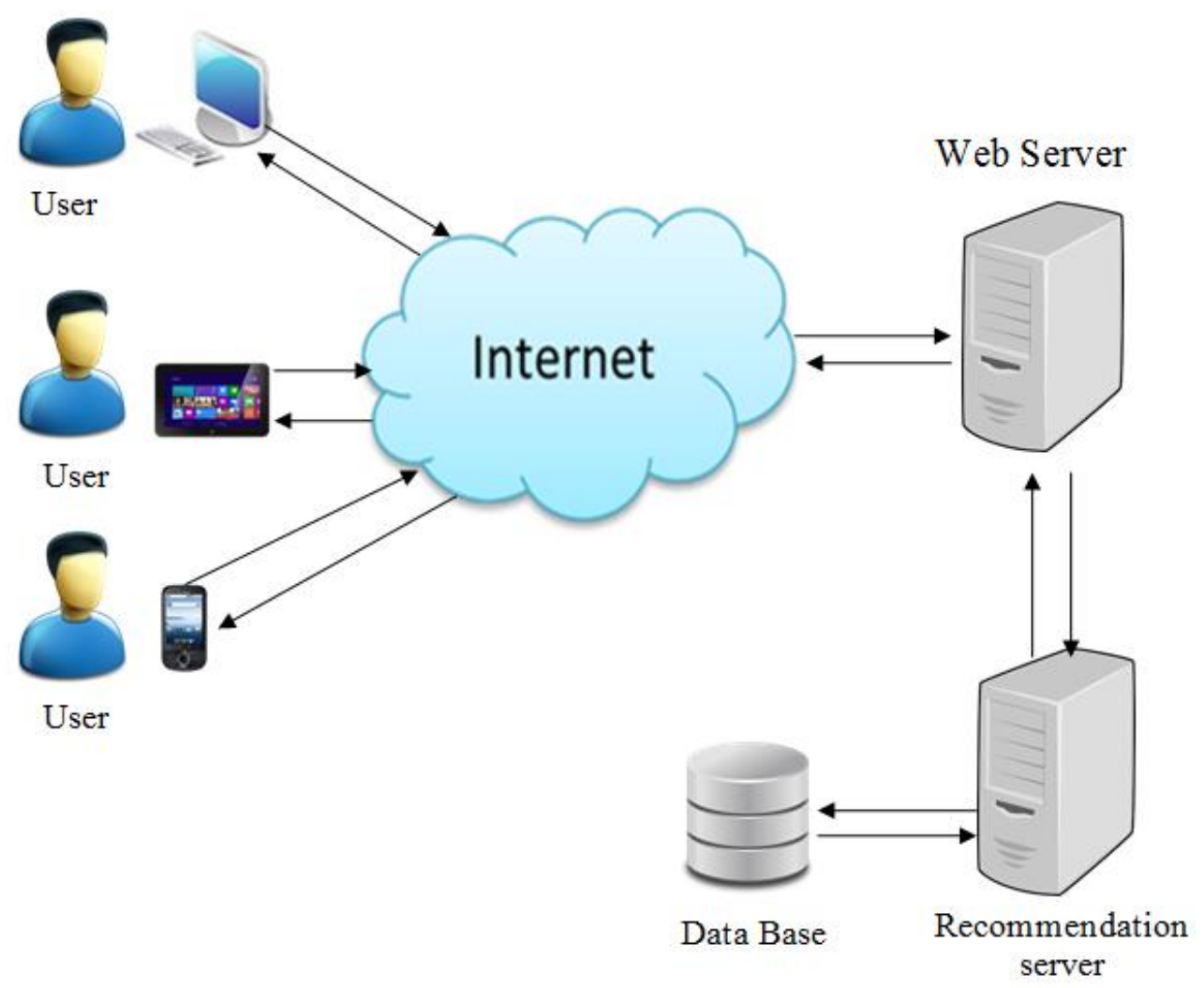

Fig 1: TFR Outline

\section{THE PROPOSED SYSTAM}

In this section, a food recommender system is presented for tourists called TFR. TFR identifies the user's interests to help them choose their favorite food in the country they have traveled to. TFR is web-based in order to enable the users to connect to the system via internet using any available devices around them with no need to install any software. Figure 1 shows the general diagram of TFR.

As showed in Figure 1, the system includes these parts:

- User: The users could use the system by connecting to the internet (mobile, pc, tablet, etc.); they register their interests in the system and receive necessary recommendations.

- Web server: The web server task is to receive and transfers information through the internet. In this part, information is sent to the recommender server for analysis and after the actions has been done in the recommender server, the web server reply back the information to the users via internet.

- Recommender server: The recommender server assesses the received information from the web server and the registered preferences in data base for each user then send suitable recommendations to the web server prioritizing them via the recommending algorithm.

- Data base: The users' preferences and feedback is recorded in data base in order to be used to give later recommendations. Users wouldn't need to enter their primary information but they could change them.
The overall process of giving recommendation by TFR is shown in Figure 2. The users who work with the system for the first time, should register in the system and do primary steps (As detailed in section 3.1). Then the user sings in to the system, and enters the necessary information. Adoptive recommending algorithm (introduced in 3.2) prioritizes the recommendations and presents them to users based on received information. TFR checks to see whether any feedback from recommendations has been received from users or not (Stated in 3.4). If the user did not give any feedback to the system for any reason, the user is asked to enter necessary information in the system.

\subsection{Primary Stages for New Users}

As shown in Figure 2, the new user must register in the system. Each user is recognized by his/her unique user name. Next steps are includes the user's unfavorable items, filling out special disease form, and determining their taste.

\subsubsection{Determining User's Unfavorable Items}

We should mention that one of the problems should be pointed out is receiving the user's primary information. Here, there are two problems:

Asking user to enter lots of information needs the user's lots of effort; therefore, the user will be disappointed and maybe leave the system.

If the primary information is little (not enough), the system does not have enough information about the user; therefore, the system present recommendations that may not be perfect for the user [19].

To solve the stated problems, the following procedure is used: 


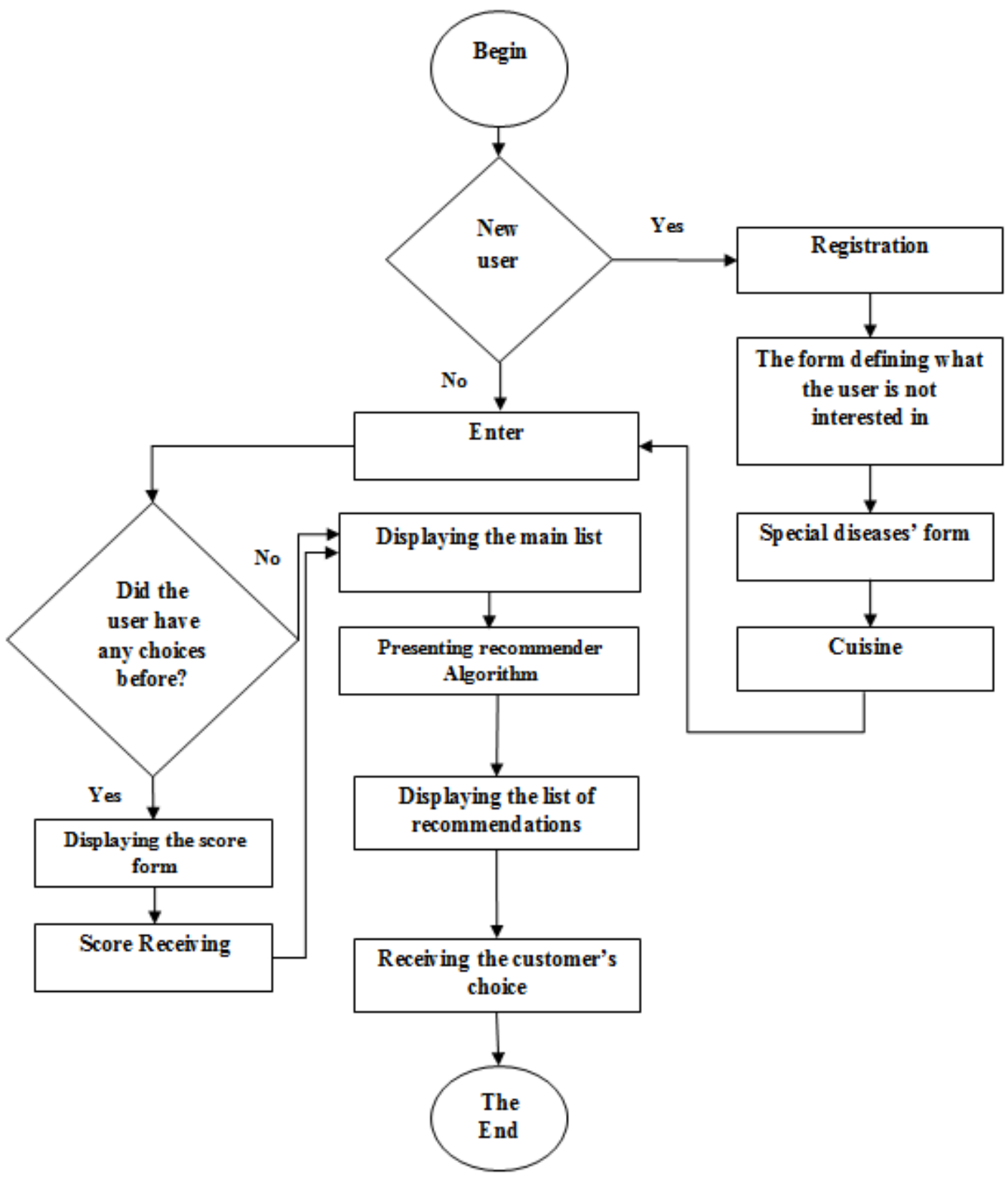

Fig 2: TFR Flowchart

For example in TFR, asking questions such as "what ingredients do you like?", the first stated problem occurs and the user may be forced to enter lots of information and this results in getting disappointed about the system. In order to solve this problem, at first when the user enters the system, is asked "which ingredients don't you like?" because the scope of the foods most people do not like is smaller than what they like. That's why when the user defines what kinds of food he/she does not like, the presented system which works based on scoring, reduces those kinds of food's scores to lessen their selection probability for the target user.

\subsubsection{Special Diseases Specification}

Next step in figure 2 is to fill out a form about special diseases. This form reduces the probability of choosing harmful foods for the user. This is the procedure: the user specifies his diet or disease (same as specifying unfavorable foods), and then TFR remarkably reduces harmful food ingredients, and; therefore, that food selection probability highly decreases.

\subsubsection{Cuisine Specification}

Then the system asks the user's cuisine. For instance, an Indian has an Indian cuisine but he may choose other countries' cuisine.

For example if an Indian user selects Italian cuisine, he specifies for the system that he prefers to eat what Italians prefer. Then the user could sign in to the system and receive the necessary recommendations. There is no need to do these primary steps by the user any time using the system. (But he/she could change them if he/she likes).

After the user enters the system, he/she is asked to specify the current country. Since the system is implemented for tourism industry, the names of twelve touristy countries of the world in 2013 in company with Iran are listed. The user specifies the country which he/she is in and likes to receive the food 
recommendation according to that country. Actually this list is expandable and the name of other countries can be added later.

\subsection{Adoptive Recommending Algorithm}

Adoptive algorithm is seen more and more in recent researches. Adoptive algorithm parameters are regulated automatically based on statistics and fixed conditions. They may change according to conditions [30, 31]. Two algorithms are used in TFR in order to increase users' ability in choosing foods. The first one is Neighbor-based Adoptive Recommender Algorithm abbreviated to NRec (Neighborrecommend). The next algorithm is the User's interests-based Recommender algorithm called URec (User-Recommended). The user can select one of the algorithms.

\subsubsection{Neighbor-based Adoptive Recommender Algorithm (NRec)}

Since at first the system does not have accurate information about the user's personal interests, that's why the system uses some other users' information who have the same interests as this user to present more accurate recommendations. Figure 3 shows the Nrec algorithm.

In TFR, some users are identified as the target user's neighbors. That should be common in cuisine and disease. If the users are similar in one parameter (cuisine or disease), they are not recognized as neighbors. Because if only, cuisine is considered it is possible for the system to recommend harmful foods for the user. Moreover if only, disease parameter is considered the foods are recommended to the user may not match his/her cuisine.

After finding the neighbors, the foods recommended to them and they scored them, will be extracted from database. To compute the score of each recommended food, the number of times they were selected by the neighbors is extracted from database. If the user has several neighbors selecting these foods, the selection times are added to each other. Then the system checks to see whether at least the score of 10 ingredients in the target users' data have reached to 75 or not. There are two important points should be mentioned here:

To extract the necessary numbers of the algorithm, first the most famous dishes of thirteen touristy countries were collected and studied. After some studies, it is found that the dishes including 5 to 15 ingredients; therefore, 10 is used that is the average of the ingredients of a dish is used.

Since ingredients' scores in TRF system is considered between 0 and 100; after doing some research in our opinion, if at least the score of 10 ingredients reaches to 75 in the target user's list, the system has got expert in the user's interests.

According to the two mentioned cases if 10 ingredients' score reaches to 75 , it means the system is more aware about the users' interests; therefore it is more rational that in this situation, the user's own proportion for selection increases and the neighbor's score proportion gradually decreases.

Therefore, variant $\alpha$ in NRec equation decreases and variant $\beta$ in recommender equation increases based on the user's own interests (URec). If ten user ingredients' score do not reach to
75 , the recommender equation according to the neighbors is as Equation 1.

$$
X=\alpha \times \boldsymbol{A}
$$

Where $\mathbf{A}$ is the number of times a food has been selected by neighbors and there will be no change in $\alpha$. Here, it should be mentioned that a differential average is used in NRec and URec. According to this method, overall, the sum of $\alpha$ and $\beta$ equal to 1 , and this stays constant even if $\alpha$ and $\beta$ change.

Since the considered variant for the two proposed equation is between 0.1 and 0.9 on the basis of neighbors' and recommendation based on the user's own interest; therefore, any time the user asks the system for a recommendation, if 10 ingredients' score by the user reaches 75 , the system subtracts 0.1 unit from variant $\alpha$. (This continues till it reaches to 0.1 ) and the system adds 0.1 unit to $\beta$ until it reaches 0.9 . Of course if the user's interests change, for instance, the user catches an illness and makes some general changes in the system by choosing a special diet, and these changes result in a situation that the system cannot identify the user's interests and to present better recommendations needs to use the neighbors' information, it is possible that $\beta$ decreases until the system finds enough information about the user again.

\subsubsection{User-based Adoptive Recommender} Algorithm (URec)

The first stages of URec algorithm is the same as NRec and is common for both methods. The same as NRec algorithm after the user specifies the target country, at first that country's foods and then their ingredients are checked by TFR as like as $\mathrm{NRec}$ algorithm. If the score of 10 ingredients in the user's data in the target user's chart reaches to 75 , then 0.1 unit is added to $\beta$ in URec until $\beta$ reaches to 0.9 . If the score of 10 ingredients do not reach to 75 , it means the system did not find complete knowledge about the user' interests; therefore, $\beta$ remains as the primary level of 0.1 . (At the beginning of working with the system, since the knowledge is the least, therefore; the smallest amount is given).URec relation is as Equation 2.

$$
Y=\beta \times \boldsymbol{B}
$$

Where, $\mathbf{B}$ is the Food's ingredients' average score in the target countries.

\subsubsection{Displaying List of Recommendations}

As mentioned earlier, in TFR it has been tried to present recommendations that are not sheer recommendations to give the user more chance to select; therefore the presented recommendations are presented into two recommender groups. One of them is neighbor-based recommender group and the other one is user interests-based recommender group. In these two groups, the target country's foods are ordered according to their scores decreasingly. In any methods, in TFR view, the food which is in the highest place and has the highest score, matches mostly to the user's interest, but for any reason (lack of chosen food, high prices, and etc.) the user may want to choose second or third food or any other food having lower score than the first recommended food from the list. 
1. Finding the users with the same cuisine as the target user.

2. Finding the users with the same diet as the target user.

3. Associate step 1 and step 2 and finding the neighbors.

4. Finding the foods previously recommended to the neighbors in that country.

5. Work out the times the neighbors have selected the foods in stage

4.

6. Add the times the neighbors have selected the same food.

7 .The system checks to see whether at least, the score of 10 ingredients in the target user's list gets to 75 or not?

8. If the result of stage 7 is negative, the recommendations based on NRec will be obtained as Equation 1.

9. The quantity of primary variant $\alpha$ equals to 0.9 . If the result of stage 7 is positive, the system decreases variant $\alpha$ to 0.1 any time the user uses the system. The system gives the necessary recommendations after computing the foods' scores.

10. Displaying the list of foods in order of scores from stages 8 and 9 .

Fig 3: NRec Algorithm

\subsection{Users' Feedback}

The users are asked to score the selected food after the system receives the user's selections. If for any reason, the user does not score the selected food, next time when the user wants to use TFR (as showed in Figure 2) first it will be checked if the user had any selections before or not? If the user had any selections before, and he did not score them, the score sheet is shown in order for the user to score the selected food.

\subsection{Score-Recording Method}

The users' feedback will be recorded at the system for later decisions after they choose a kind of food and they score it. In here two points are important to be mentioned:

These received scores are just based on the user's cuisine. The user, who records this score in the system, does not pay attention to the other features the food may have (such as cost, nutrient, etc.). TFR has been designed to just base on the user's cuisine and interests.

It is assumed in TFR that all food eaten by the user, are the ones cooked based on standards, and if a kind of food is not up to standard because of some changes for improvement or a better taste, that food will not be recorded.

The scores that the users record in the system for each kind of food are between 1 and 5. Any score is given to a kind of food by the user; the score of that food's ingredients becomes two times bigger. For example, if a user eats food A that contains ingredients $\left(X_{1}, \ldots, X_{n}\right)$ and scores that food 3, then 6 units are added to each ingredient $\left(X_{1}, \ldots, X_{n}\right)$ systematically if another user eats B and scores that food 5, 10 scores are added to each ingredient of B. In later stages to propose recommendation to the users, the higher is the score of a group of ingredients, the more probable is that the food containing these ingredients be chosen.

\section{EXPERIMENTS AND RESULTS}

In this part, it is tried to do some experiments to evaluate TFR. In order to do this, the proposed system is implemented on collaborative and content-based filtering methods to check which method is better for the implemented system. After comparing these two methods, the TFR system is implemented based on filtering method which has more efficiency.

To evaluate the accuracy of the recommended algorithm, precision and recall that are two common metrics for measuring the accuracy of recommender algorithm are used. These metric methods are used to identify the best filtering method and in another case, they are used to evaluate TFR.

The heading of a section should be in Times New Roman 12point bold in all-capitals flush left with an additional 6-point of white space above the section head. Sections and subsequent sub- sections should be numbered and flush left. For a section head and a subsection head together (such as Section 3 and subsection 3.1), use no additional space above the subsection head.

\subsection{Equalization}

In TFR, some ingredients have various kinds. For example, all kinds of oyster or lobster are classified in one group because many users such as Iranians probably do not have any knowledge about different kinds of lobsters or oysters, and using different words that refer to the same ingredients and just a slight difference in kind, confuses the user more. In addition to these, the items, which do not have any effect on the system's recommending function, have been equalized. For example different colors of bell pepper is used in different foods used more for food dressing and has no effect on the quality of given recommendations by TFR. Table 1 shows the equalization of some foods that has been used in TFR. 
It is necessary to be mentioned that by equalization, the options which could be selected by the user are declined and decrease the user's attempt to enter primary information and so it will able the system to identify the user's preferences and interests faster.

\subsection{Implementation}

To assess the function of proposed system in this part, at first collaborative and content-based filtering methods are compared. Then the system's function is checked and precision, recall and $\mathrm{F}$ measure are computed.

\subsubsection{Comparing Collaborative and Content-}

\section{Based Filtering Methods}

120 users have been used to compare the collaborative filtering and content-based methods. The users had different educational degrees and, they were 18 to 68 years old. For the comparing activities, the presented system in this stage is limited to a smaller system. The implemented system just includes Iranian foods, but all the same ingredients considered for the smaller system as for the general system. All users have enough information and knowledge about the proposed foods. The aim of this stage is just prioritizing the food and recommends them based on the information about the users. Normally the first recommended food in the recommendations list is the food anticipated by the system to be the most similar to the user's cuisine. After the recommendation presented, the difference between the first recommended food by the system and the food selected by the user is computed.

\subsubsection{Implementing TFR Based On Content-}

\section{Based Filtering}

At first the system was implemented based on content-based filtering method. As mentioned earlier, when a system is content-based, neighbors' information is not used. After the system was implemented, each user was asked to work with the system. First each user chooses the ingredients that he/she does not like (he/she is not interested in). Then a list of Iranian foods which the user knows well is shown. It is necessary to be mentioned the system prioritizes the recommended foods in the list of recommendations based on the information received from the user up to now. Then the user chooses a food matching his interests more. In this stage it is important for us which food in the list the user selects because nearer the selected food by the user to the first recommended food by the system; the more accurate is the system.

Table 1: Equalizations of Foods in TFR

\begin{tabular}{|c|c|c|c|}
\hline $\begin{array}{l}\text { General } \\
\text { Equal }\end{array}$ & First Case & Second Case & Third Case \\
\hline Bell Pepper & $\begin{array}{ll}\text { Red } & \text { Bell } \\
\text { Pepper } & \end{array}$ & $\begin{array}{l}\text { Yellow Bell } \\
\text { Pepper }\end{array}$ & $\begin{array}{ll}\text { Green } & \text { Bell } \\
\text { Pepper } & \end{array}$ \\
\hline Crab & Lobster & Crab Tail & -- \\
\hline Wine & Red Wine & White Wine & Rice Wine \\
\hline Fish & Salmon & Red Snapper & Mackerel \\
\hline Shrimp & Prawn & -- & -- \\
\hline Mussels & Oyster & Clam & Shellfish \\
\hline Orange & $\begin{array}{l}\text { Orange } \\
\text { Juice }\end{array}$ & -- & -- \\
\hline Lemon & $\begin{array}{l}\text { Lemon } \\
\text { Juice }\end{array}$ & -- & -- \\
\hline Pork & Ham & Lard & -- \\
\hline
\end{tabular}

\subsubsection{Implementing TFR System Based On Collaborative Filtering}

After the previous stage, the presented system changed to the condition using collaborative filtering. Now again the users are asked to work with the system. Since the user' preferences have already been recorded in the system, it is not necessary for the users to specify the ingredients they are not interested in because the system is collaborative filtering-based implemented (Is implemented based on collaborative filtering; therefore, neighbor concept is used). In this stage it is tried to find the neighbors with the same interests as the user, and give more accurate recommendations based on the users' interests.

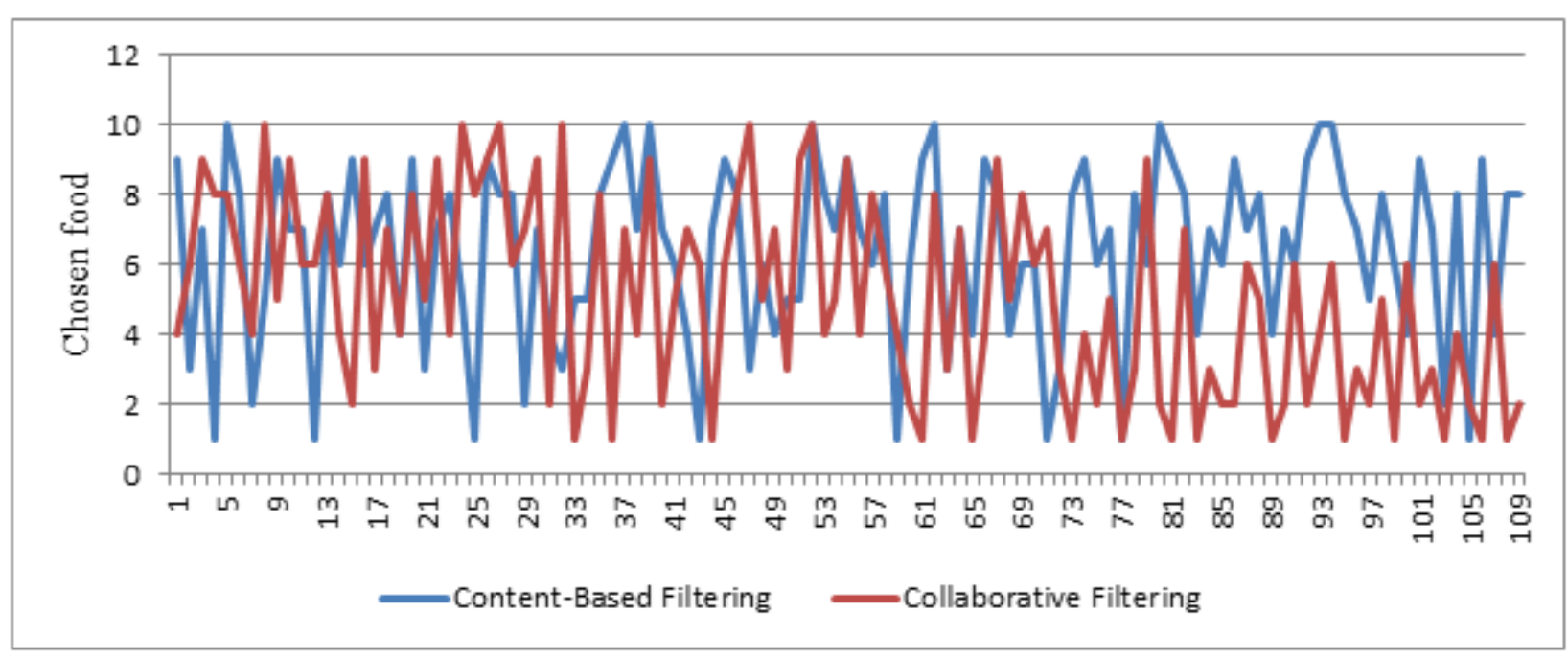

Fig 4: Nrec Algorithm Evaluation Results 


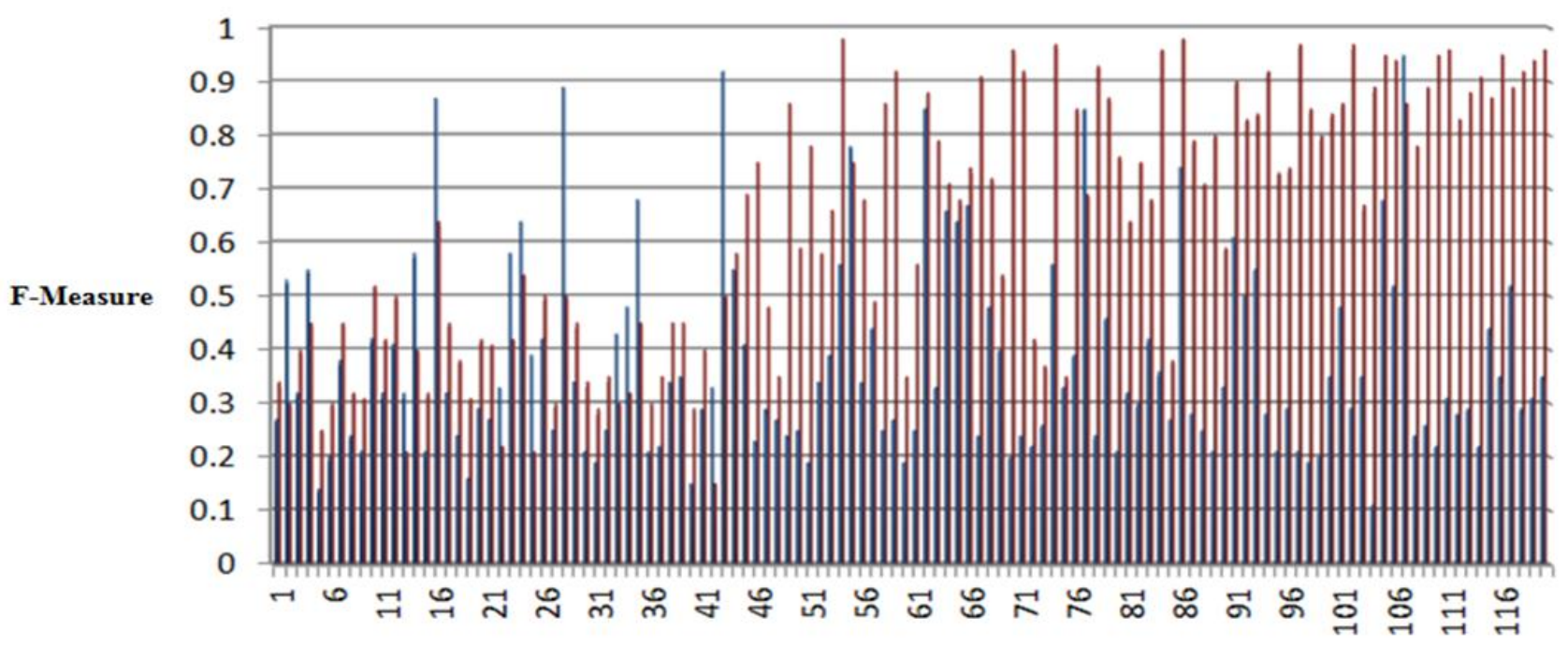

Users

\section{Content based Filtering $\square$ Collaborative Filtering}

Fig 5: Comparing the collaborative and content-based filtering methods

\subsubsection{Comparing Two Filtering Methods}

In Figure 4 and Figure 5the results of collaborative and content-based filtering methods are shown. In Figure 4, the left part of the chart is numbered 1 to 10 indicating 10-top foods recommended to the user. As mentioned, foods are arranged according to their scores, from the highest score to the lowest score. The 10th food is the best food recommended to the user. The blue lines connect the user's selecting points. These points indicate the user's selection in TFR system. As seen in the Figure 3, the content-based method is not much able in presenting suitable recommendations to the users, and very few users selected the system's best recommended foods (between 8 and 10),which are among the high-score foods. Also, Figure 5 shows the results of methods' evaluation based on their final F-Measure. As it shows, the collaborative filtering methods' results are higher when the number of users increases. In other words, by increasing number of TFR users, the collaborative filtering method performs better that the content-based filtering method.

\subsubsection{Comparing the filtering methods using evaluation metrics}

After measuring precision and Recall, the F-Measure metric is computed for each of the collaborative and content-based filtering. It is necessary to mention that for each of the 120 users, first the precision and then Recall are computed. After computing these amounts, the F-Measure metric is computed for each user in both presented methods. The results are seen in the Figure 4.It is concluded from previous steps that the collaborative filtering method in the presented system can notably improve the system's efficiency compared to the content-based method. That's why for the system's test stage, explained in the next section, the general system uses the collaborative filtering method.

\subsection{Evaluation Results}

\subsubsection{Data Set}

To the best of our knowledge the presented system is one of the first in its kind, there was no available dataset to test it; therefore, to check the efficiency of TFR, it is necessary for some users to work with the presented system in order to check the amount of efficiency in presenting recommendations. That's why 300 users with different educational degrees were asked to work with the system. Each of the stated users received recommendations from the system 6 times; therefore, there are totally 1800 valid data.

\subsubsection{Experimental Results}

From among all existing data, $70 \%$ were randomly selected as train data, and 30\% were chosen as test data. Then among 300 users, 210 users were used for training and 90 users were used for the system testing. In the training stage, the data were used to assign values to the system parameters. In the test stage, to obtain the accuracy of the system, first it is checked to see which food is selected by each of the 90 users from the recommended foods list, and what score they gave to the selected food. Since the presented system considers a score for each food, the obtained score differential by the system and the score given to that food by the user is computed for each 90 users, and then they are added to each other. The result is divided by the number of the users. The quotient indicates the system standard deviation. 


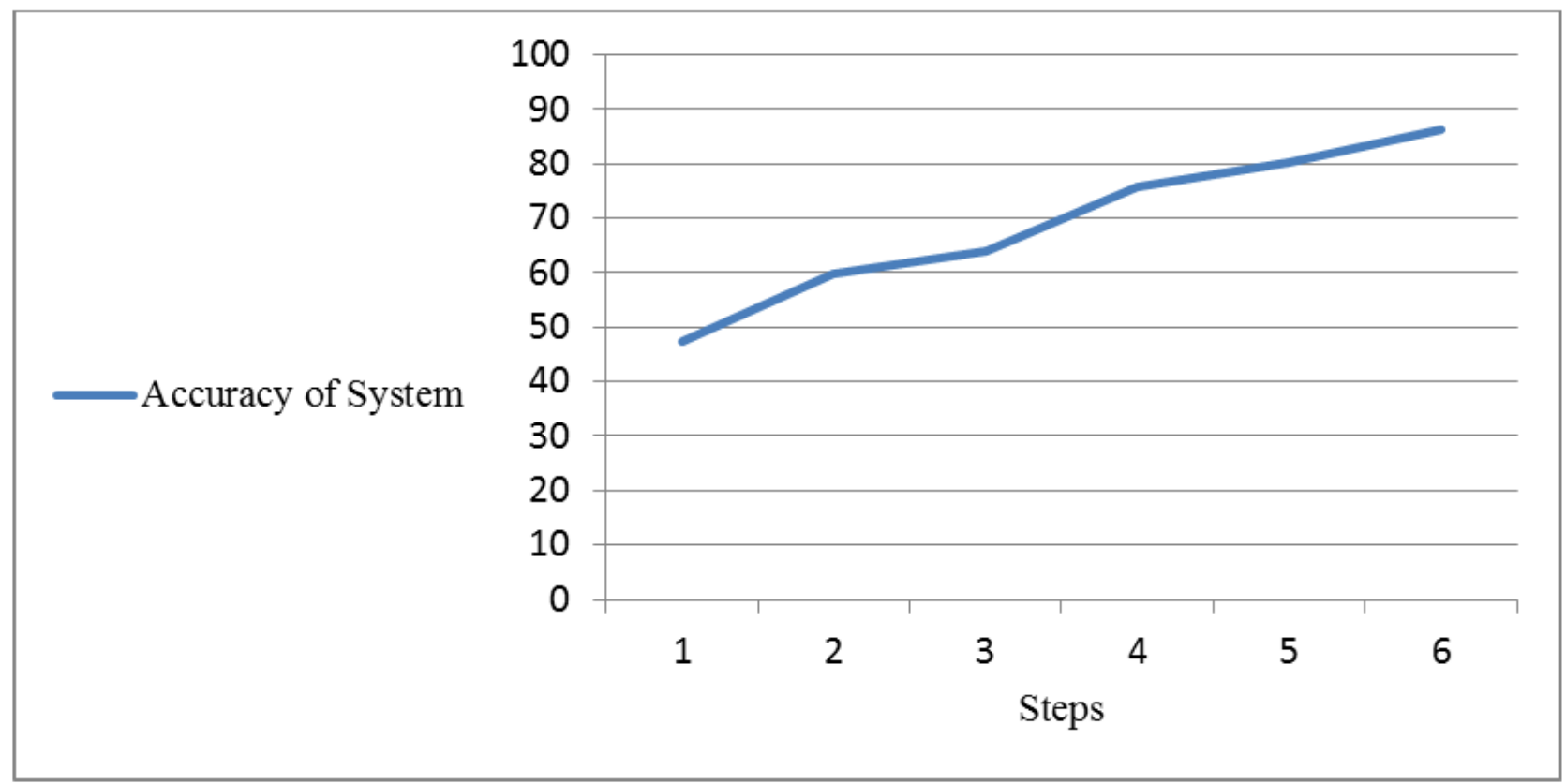

Figure 6: Accuracy of TFR in 6 Steps

Since the users' score is between 2 and 10, and it's added to the ingredients' score each time according to the users' interests, this ranges from -100 to +100 . In order to balanceboth are normalized into [0...1] by using the Equation 3.

$$
\frac{A-A_{\text {min }}}{\mathrm{A}_{\max }-\mathrm{A}_{\min }}
$$

Where, $\mathrm{A}$ is the number which is going to be normalized $A_{\min }$ is the numbers' minimum and $A_{\max }$ is the numbers' maximum.Figure 6 shows the accuracy of the system in different uses. As it shows, the accuracy increases as the user uses the system more.

\section{CONCLUSION}

In this paper, a recommender system for tourism industry has been proposed. Since the people who travel to other countries, are not much familiar with that country's foods, this system's aim is to recommend food to tourists according to their preferences. In the presented system, the collaborative and content-based filtering methods were studied, and then because of the collaborative method's better results, the presented system was implemented based on this filtering method. The system was checked by some users to be evaluated by empirical experiments. Moreover the precision and recall metrics, which are common metrics in measuring the integrity of the recommendation algorithm, were used to evaluate the integrity of recommended algorithm. These metrics were used to identify the best filtering method and also to evaluate the presented system. Finally the system was checked by 300 users that each receives some recommendations from the system 6 times. The results show TFR present recommendations to the accuracy of $86 \%$.

\section{REFERENCES}

[1] Efraim Turban, Jae Lee, David King, and H. Michael Chung. Electronic Commerce: A Managerial Perspective. Prentice Hall, 2000.
[2] Maria S.Pera,Y-Kai .N (2013). A group recommender for movies based on content similarity and popularity .information processing and management , 673-687.

[3] Ingrid A.Christensen, S. S. (2011). entertainment recommender system for group of user. expert systems with applications , 14127-14135.

[4] Joseph A. Konstan, Bradley N. Miller, David Maltz, Jonathan.L. Herlocker, Lee R. Gordon, and John Riedl 1997.GroupLens: Applying collaborative filtering to Usenet news.ommunications of the ACM, 40(3): pp 7787.

[5] M. Tavakolifard, J. Gulla, K. Almeroth, J. Ingvaldesn,G. Nygreen, and E. Berg. Tailored news in the palm of your hand: a multi-perspective transparent approachto news recommendation. In Int. Conf. on WorldWide Web, pages 305-308, 2013

[6] konstantinoschristidis,gregorisMentzas. (2013). a topicbased recommender system for electronic marketplace platforms. expert systems with applications , 4370-4379.

[7] José M. Noguera, Manuel J. Barranco, Rafael J. Segura, Luis Martnez. (2012). A mobile 3D-GIS hybrid recommender system for tourism. Information sciences, pages 37-52.

[8] Wan-Shiou Yang, San-Yih Hwang (2013), iTravel: A recommender system in mobile peer-to-peer environment. The Journal of Systems and Software, 1220 .

[9] Chieh-Yuan Tsai, Shang-Hsuan Chung, (2012) A personalized route recommendation service for theme parks using RFID, Decision Support Systems, 514-527.

[10] Duen-YianYeh, Ching-Hsue Cheng, (2015), Recommendation system for popular tourist attractions in Taiwan, Tourism Management, 164-176.

[11] http://en.wikipedia.org/wiki/World_Tourism_rankings 
[12] M. Svensson, K. hook, R. Coster, 2005, Designing and Evaluating Kalas: A Social Navigation System for Food Recipes, ACM Transactions on Computer-Human Interaction, Vol. 12, No. 3, Pages 374-400.

[13] M. Svensson, K. Höök, J. Laaksolahti, A. Waern, Social Navigation of Food Recipes, 2001, Proceeding of the SIGCHI, ACM

[14] Jong-Hun Kim, Jung-Hyun Lee, Jee-Song Park, YoungHo Lee, Kee-Wook Rim (2009) Design of Diet Recommendation System for Healthcare Service Based on User Information International Conference on Computer Sciences and Convergence Information Technology. IEEE, pp. 516-518.

[15] Xiaocheng Li, Xinliu, Zengjie Zhang, Yongming Xia, SongrongQian (2010) Design of Healthy Eating System based on Web Data Mining, WASE International Conference on Information Engineering. IEEE, 346-349

[16] Giuseppe Di Fabbrizio, Narendra Gupta, SvevaBesana, Premkumar Mani, (2010), Have2eat: a Restaurant Finder with Review Summarization for Mobile Phones, pp 1720

[17] J. F. McCarthy, 2002, Pocket restaurant finder: a situated recommender system for groups, Technology Labs 161 North Clark Street, Chicago, IL 60601 USA.

[18] P. Resnick, HR. Varian, 1997, a survey on recommender systems, Communications of the ACM, vol. 40, pp. 5659.

[19] M. Rashid, I. Albert, Dan Cosley, Shyong K. Lam, Sean M. Mcnee, Joseph A. Konstan, J. Riedl, 2002, Getting to Know you: Learning new user preferences in recommender systems, ACM.

[20] S. H. Ha, 2006, Digital content recommender on the internet, Intelligent Systems, IEEE, vol. 21, No. 2, pp. $70-77$.

[21] D. Li, Q. Lv, H. Xia, L. Shang, 2011, Lupistis: A privacy-preserving content recommender system for online social communities, Web Intelligence and Intelligent Agent Technology (WI-IAT), Vol.1. pp. 7986.

[22] J. L. Herlocker, J. Konstan, L. G. Terveen, J. T. Ridel, 2004, Evaluating Collaborative Filtering Recommender
Systems, ACM Transactions on Information Systems, Vol. 22, No. 1, Pages 5-53.

[23] Ji-Chun Quan, Sung-Bae Cho, A Hybrid Recommender System Based on AHP That Awares Contexts with Bayesian Networks for Smart TV, Hybrid Artificial Intelligence Systems, Volume 8480, 2014, pp 527-536

[24] A. I. Schein, A. Popescul, L. H. Ungar, and D M.Pennock. Generative models for cold-start recommendations. In Proceedings of the 2001 SIGIR Workshop on Recommender Systems, 2001.

[25] SCHEIN, A.L. POPESCUL, A. UNGAR, L.H. 2002. Methods and Metrics for Cold-Start Recommendations. In SIGIR'02, Tampere, Finland

[26] L. Safoury, A. Salah, 2013, Exploiting User Demographic Attributes for Solving Cold-Start Problem in Recommender System, Software Engineering, Vol. 1, pp. 1-22.

[27] Dong-Her Shih, David C.Yen, Ho-Cheng Lin, MingHung Shih. 2011, An implementation and evaluation of recommender systems for traveling abroad, Expert Systems With Applications, Vol.38, pp. 15344-15355.

[28] Garsia, L. Sebastia, E. Onaindia, 2011, On the design of individual and group recommender systems for tourism. expert systems with applications, vol. 38. Issue 6, pp.7683-7692.

[29] L. Martinez, R.M. Rodrigues, M. Espinilla, 2009, REJA: A Georeferenced Hybrid recommender system for restaurants. IEEE, International conference on Web intelligence and intelligent agent technology.

[30] ZiHanZhuo, WeiMingZhai, Xin Li, LingLing Liu, JinTian Tang, 2014, Local adaptive segmentation algorithm for 3-D medical image based on robust feature statistics, Science China Information Sciences, Volume 57, Issue 10, pp 1-12

[31] Gunawan,M.A.Sulaiman, F. ; Putra, T.A. ; Hasudungan, C.D. ; Sari, R.F. 2014, Object-following robot using adaptive cruise control algorithm with IOIO, Intelligent Green Building and Smart Grid (IGBSG), 2014 International Conference. IEEE. Pages 1-5. 